\begin{tabular}{|c|c|}
\hline Title & ZAPS is a potent stimulator of signaling mediated by the RNA helicase RIG-I during antiviral responses \\
\hline Author(s) & $\begin{array}{l}\text { Hayakawa, Sumio; Shiratori, Souichi; Y amato, Hiroaki; Kameyama, Takeshi; Kitatsuji, Chihiro; Kashigi, Fumi; Goto, } \\
\text { Showhey; Kameoka, Shoichiro; Fujikura, Daisuke; Y amada, Tai sho; Mizutani, T atsuaki; Kazumata, Mika; Sato, Maiko; } \\
\text { T anaka, Junji; A saka, Masahiro; Ohba, Y usuke; Miyazaki, T adaaki; Imamura, Masahiro; T akaoka, A kinori }\end{array}$ \\
\hline Citation & $\begin{array}{l}\text { Nature Immunology, 12(1), 37-44 } \\
\text { https://doi.org/10.1038/hi.1963 }\end{array}$ \\
\hline Issue Date & 2011-01 \\
\hline Doc URL & http:/hdl.handle.net/2115/46762 \\
\hline Type & article (author version) \\
\hline Additional Information & There are other files related to this item in HUSCAP. Check the above URL. \\
\hline File Information & NI12-1_37-44.pdf \\
\hline
\end{tabular}

Instructions for use 


\section{ZAPS is a potent stimulator of signalling mediated by the RNA helicase RIG-I during antiviral responses}

Sumio Hayakawa ${ }^{1,8}$, Souichi Shiratori ${ }^{1,3,8}$, Hiroaki Yamato ${ }^{1,4,8}$, Takeshi Kameyama ${ }^{1}$, Chihiro Kitatsuji ${ }^{1}$, Fumi Kashigi ${ }^{1,7}$, Showhey Goto ${ }^{1,7}$, Shoichiro Kameoka ${ }^{1,7}$, Daisuke Fujikura $^{5}$, Taisho Yamada ${ }^{1}$, Tatsuaki Mizutani ${ }^{6}$, Mika Kazumata ${ }^{1}$, Maiko Sato ${ }^{1}$, Junji Tanaka ${ }^{3}$, Masahiro Asaka ${ }^{4}$, Yusuke Ohba ${ }^{6}$, Tadaaki Miyazaki ${ }^{5}$, Masahiro Imamura $^{3}$ \& Akinori Takaoka ${ }^{1,2}$

${ }^{1}$ Division of Signaling in Cancer and Immunology, and ${ }^{2}$ Research Center of Infection-associated Cancer, Institute for Genetic Medicine, Hokkaido University, Kita-15, Nishi-7, Kita-ku, Sapporo 060-0815, Japan. ${ }^{3}$ Department of Hematology and Oncology, Hokkaido University Graduate School of Medicine, and ${ }^{4}$ Department of Gastroenterology, Hokkaido University Graduate School of Medicine, Kita-15, Nishi-7, Kita-ku, Sapporo 060-8638, Japan. ${ }^{5}$ Department of Bioresources, Hokkaido University Research Center for Zoonosis Control, Kita-20, Nishi-10, Kita-ku, Sapporo 001-0020, Japan. ${ }^{6}$ Laboratory of Pathophysiology and Signal Transduction, Hokkaido University Graduate School of Medicine, Kita-15, Nishi-7, Kita-ku, Sapporo 060-8638, Japan. ${ }^{7}$ Department of Chemistry, Graduate School of Science, Hokkaido University, Sapporo, Japan. ${ }^{8}$ These authors contributed equally to this work. Correspondence should be addressed to A.T. (takaoka@igm.hokudai.ac.jp).

The poly(ADP-ribose) polymerases (PARPs) participate in various processes. Here, we report that the PARP-13/ZAP shorter isoform (hereafter called ZAPS), rather than the full length protein, is selectively induced by 3pRNA, and functions as a potent stimulator of retinoic acid-inducible gene-I (RIG-I)-mediated interferon (IFN) responses in human cells. ZAPS associates with RIG-I to promote the oligomerization and ATPase activity of RIG-I, leading to robust activation of IRF3 and NF-אB pathways. Disruption of the PARP-13/ZAP gene, ZC3HAV1, severely 
abrogated the induction of IFN- $\alpha$, IFN- $\beta$ and other cytokines upon viral infection. These results indicate that ZAPS is a key regulator of RIG-I signaling during the innate antiviral immune response, suggesting its possible use as a therapeutic target for viral control.

Pathogen invasion is sensed by pattern recognition receptors (PRRs) of the innate immune system through the recognition of pathogen-associated molecular patterns (PAMPs). Some subsets of the PRR trigger the activation of intracellular signaling pathways leading to the induction of antimicrobial genes. The repertoire of such signal transducing-type PRRs includes membrane-bound Toll-like receptors (TLRs) and C-type lectin receptors (CLRs), as well as cytosolic receptors such as retinoic acid-inducible gene-I (RIG-I)-like receptors (RLRs) and NOD-like receptors (NLRs) ${ }^{1-3}$. Nucleic acids derived from viruses are key signatures that trigger antiviral defence against viral infection. Among them, viral RNAs can serve as a major PAMP that is targeted by TLR3 and TLR7/8 on the endosomal membrane and by RLRs in the cytosolic space.

At least three DExD/H-box RNA helicases ${ }^{4,5}$, RIG-I, MDA5 and LGP2 are included in the RLR family. RIG-I is a key PRR for the detection of positive- and negative-stranded RNA viruses in the cytoplasm of cells ${ }^{6,7}$, and plays an important role in triggering responses against many viruses, such as orthomyxovirus (influenza A virus) and paramyxovirus (measles, mumps, and Sendai virus $(\mathrm{SeV})$ ) families, hepatitis $\mathrm{C}$ virus (HCV), and Japanese encephalitis virus $(\mathrm{JEV})^{8}$. 5'-triphosphate modifications of RNA (3pRNA) are essential for RIG-I recognition and activation ${ }^{9,10}$. Ligand-binding activates the ATPase activity of RIG-I to change its structural conformation ${ }^{6}$, which in turn enables RIG-I to interact through its N-terminal tandem caspase recruitment domain (CARD) with the adaptor protein MAVS (for mitochondrial antiviral signaling protein, also known as IPS-1, VISA, or Cardif) ${ }^{11-14}$. MAVS then initiates the activation of interferon (IFN)-regulatory factor (IRF) 3, IRF7 and NF- $\mathrm{B}$ transcriptional pathways for the subsequent production of type I IFNs and inflammatory cytokines, which are crucial for activating innate immune responses to viral infection ${ }^{6,15}$. Given the important role of the RIG-I pathway in the antiviral innate response, the mechanisms regulating RIG-I activation represent a topic of intense research ${ }^{16-18}$.

Poly(ADP-ribose) polymerases (PARPs), a superfamily with least 17 members, are known to regulate not only cell survival and cell death programs triggered by DNA 
damage, but also other biological functions as well as pathological processes, such as inflammatory and degenerative diseases, in a manner dependent or independent of their PARP activity ${ }^{19-22}$. Several PARP-superfamily members have a direct regulatory effect on replication of certain viruses ${ }^{19,20,22,23}$. For example, PARP-1 directly poly(ADP-ribosyl)ates Epstein-Barr virus (EBV)-encoded origin binding protein EBNA1 or Kaposi's sarcoma-associated herpesvirus (KSHV)-encoded latency-associated nuclear antigen LANA, and disables their functions ${ }^{23,24}$. PARP-13 (also termed ZAP; zinc-finger CCCH-type antiviral protein 1) interacts with viral RNAs from certain subsets of viruses, such as Moloney murine leukemia virus (MLV) and Sindbis virus (SIN), and recruits the RNA exosome and the p72 DEAD box RNA helicase to efficiently degrade RNAs ${ }^{20,25,26}$.

PARP-superfamily members also contribute to the host inflammatory and immune responses to viral infection. PARP-1 functions as a co-activator of NF- $\mathrm{B}^{27,28}$ a function for which the PARP enzymatic activity does not seem to be required. PARP-9/BAL1, which lacks PARP activity ${ }^{29}$ and is inducible by IFN- $\gamma$, is able to increase the expression of IFN-stimulated genes ${ }^{30}$, suggesting a putative role in host defence against viral infection. However, the role of PARP-superfamily members in the activation of innate signaling pathways mediated by PRRs such as RIG-I is not clearly understood.

Here, we show that the shorter isoform of PARP-13/ZAP (ZAPS; ZAP, short form) rather than the full length isoform, is selectively induced by 3pRNA, and functions as a regulator of RIG-I-mediated signaling. Knockdown experiments revealed that ZAPS plays a role in type I IFN gene induction in various human cell lines and human primary cells. Mechanistically, ZAPS directly associates with RIG-I in a ligand-dependent manner to efficiently promote the oligomerization of RIG-I, resulting in a marked increase in its ATPase activity and subsequently enhancing the activation of the downstream IRF3 and NF- $\kappa$ B signaling pathways. Consistent with these results and zinc finger nuclease (ZFN)-based gene disruption data, ZAPS strengthens RIG-I-mediated induction of type I IFNs and other inflammatory cytokines, and inhibits viral replication upon infection with RNA viruses that involve RIG-I, such as influenza virus and Newcastle disease virus (NDV). These findings indicate that ZAPS has an important role as a potent stimulator of RIG-I signaling in the innate immune response to viral infection.

\section{RESULTS}




\section{PARPs contribute to the IFN response}

To investigate the role of the PARP-superfamily members in nucleic acid induced innate immune responses, we selected some of the PARP-superfamily members known to be involved in microbial infection, inflammation and immunity: PARP-1, PARP-2, PARP-7, PARP-9, PARP-12 and PARP-13 (refs. 19,23,31-34). We then examined whether they have the ability to enhance the induction of IFNB mRNA in HEK293T cells in response to stimulation with three different types of nucleic acids- 3pRNA, poly(rI:rC) and poly(dA-dT)•poly(dA-dT) (named poly(dA:dT) hereafter).

Among the protein tested, PARP-13 uniquely showed a marked enhancing effect on IFNB mRNA expression induced by stimulation with 3pRNA, poly(rI:rC) and poly(dA:dT) (Fig. 1a), all of which are known to activate the RIG-I-mediated pathway in HEK293T cells ${ }^{9,10,35-37}$. A weak increase in IFNB mRNA expression was also detected in cells expressing PARP-1, PARP-2 and PARP-9. PARP-13 exists in at least two isoforms ${ }^{22,26,38}$. The amino-terminal 254-amino acid fragment of the rat homologue, which corresponds to the N-terminal one-third portion of the shorter isoform of human PARP-13 protein, was previously identified as rat $\mathrm{N}$-terminal zinc finger antiviral protein $(\mathrm{rNZAP})^{20}$. The expression of rNZAP results in a specific decay of viral mRNAs without any effect on the levels of mRNAs derived from host cells ${ }^{20,39}$. However, the role of human PARP-13 in type I IFN induction remains unknown.

We first examined the role of the two PARP-13 isoforms in the induction of type I IFN by 3pRNA. Both isoforms can enhance IFNB mRNA induction and IFNB promoter activation following 3pRNA stimulation (Fig. 1b). However, the shorter form of PARP-13 (isoform 2; ZAPS), which lacks the PARP domain, has a stronger effect than the full-length PARP-13 (isoform 1; ZAP) (Fig. 1b). This indicates that IFN induction does not necessarily require the PARP domain of PARP-13. Consistent with this observation, ZAPS mRNA is exclusively induced by stimulation with 3pRNA both in HEK293T cell and human primary $\mathrm{CD} 14^{+}$monocytes purified from peripheral blood mononuclear cells (PBMCs), whereas the full-form ZAP is constitutively expressed but barely induced (Fig. 1c, left panels and Fig. 1d). The promoter region of the ZAP gene contains ISRE/IRF-E elements ${ }^{40}$. We investigated if expression of ZAPS and ZAP are regulated via similar mechanisms. Treatment with IFN- $\alpha$ resulted in the preferential induction of ZAPS but not the full-form ZAP (Fig. 1c, right panels). These data suggest a distinct mechanism of ZAPS induction in comparison to ZAP, and imply a distinct role for 
ZAPS as a regulator of innate immunity. Therefore, we focused on the functional role of ZAPS in RIG-I signaling.

\section{ZAPS enhances RIG-I-mediated type I IFN production}

Next, we examined the effect of ZAPS in the RIG-I-mediated type I IFN induction. Expression of ZAPS in HEK293T cells markedly enhanced 3pRNA-induced expression of IFNA1 and IFNA4 as well as IFNB (Fig. 2a). The enhancement of IFNB induction correlated positively with ZAPS overexpression (Fig. 2b). Consistent with this result, 3pRNA induced IFNB promoter activation in a manner dependent on the amount of ZAPS expression in HEK293T cells (Fig. 2c and Supplementary Fig. 1a). Activation of type I IFN genes and IFN-related genes was observed even in the absence of 3pRNA stimulation (Fig. 2b,c and Supplementary Fig. 1b). On the other hand, ZAPS does not show any positive effect on an E2F-responsive or SRE-driving luciferase reporter (pE2F-Luc or pSRE-Luc) (Supplementary Fig. 1c) or on the induction of their related genes (Supplementary Fig. 1b).

To further evaluate the contribution of ZAPS in the RIG-I signaling, we used an RNA interference approach. Notably, all three differently targeted ZAPS small interfering RNAs (siZAPS1, siZAPS2 and siZAPS3) strongly knocked down the endogenous protein and mRNA levels of ZAPS (Supplementary Fig. 2a,b), resulting in a significant suppression of 3pRNA-induced IFNB mRNA expression in HEK293T cells (Fig. 2d, left panel). Knockdown with siZAPS3 also resulted in significant inhibition of IFN- $\beta$ production in response to 3pRNA stimulation, as assayed by ELISA (Fig. 2d, right). Furthermore, the knockdown effect of siZAPS3 could be rescued by the expression of a mouse ZAPS that does not carry the target sequence for siZAPS3 (Fig. 2e), but not by the expression of another PARP-superfamily member, PARP-1, which does not significantly affect 3pRNA-induced expression of IFNB (Figs. 2e,1a). We also examined the effect of siZAPS3 knockdown in other cell types, namely human lung epithelial adenocarcinoma cell line A549, cervical cancer cell line HeLa, and acute monocytic leukemia cell line THP-1. IFNA and IFNB mRNA induction in response to 3pRNA or viral RNAs derived from influenza virus (flu vRNA) was abrogated by endogenous ZAPS protein deletion using siZAPS3 (Fig. 2f and Supplementary Fig. 2c,d; data not shown). Furthermore, type I IFN response to 3pRNA was significantly suppressed by knockdown of ZAPS in human primary CD14 ${ }^{+}$monocytes (Fig. 2g) and fibroblasts (Fig. 2h), implying the 
physiological significance of ZAPS in human cells. These results indicate that ZAPS is a potent stimulator of the RIG-I-mediated type I IFN response.

\section{ZAPS facilitates NF- $\kappa$ B and IRF3 signaling}

We next investigated whether ZAPS can enhance NF- $\kappa$ B and IRF3 signaling downstream of RIG-I. The induction of other cytokines, such as TNF, IL6 and CXCL10 in response to 3pRNA stimulation was diminished in ZAPS-knocked down A549 cells (Fig. 3a). This suggests that ZAPS also activates the NF- $\kappa$ B pathway, another important signaling branch of the RIG-I-mediated pathway. Consistent with this idea, ZAPS overexpression in HEK293T cells induced the robust activation (13-fold) of an NF- $\mathrm{BB}$-responsive promoter following 3pRNA stimulation (Fig. 3b). However, ZAPS had a lower activatory effect on the NF- $\mathrm{B}$-responsive promoter following R848-mediated stimulation of TLR8 (Fig. 3c). 3pRNA-induced dimerization of IRF3, which is an essential transcriptional factor for RIG-I-mediated type I IFN production, was significantly reduced in siZAPS3-pretreated HEK293T cells (Fig. 3d). In addition, electrophoretic mobility shift assay revealed that NF- $\mathrm{B}$ activation in response to 3pRNA stimulation was inhibited in siZAPS3-pretreated HEK293T cells (Fig. 3e). Collectively, these data indicate that ZAPS may be an essential component of the RIG-I-mediated signaling pathway, affecting the activation of both the IRF3 and the NF- $\kappa$ B activation pathways.

Activation of RIG-I transmits signals to the adaptor protein MAVS, leading to the activation of TANK-binding kinase 1 (TBK1), which is one of the IRF3 kinases ${ }^{6,7}$. STING (stimulator of interferon genes, also known as TMEM173, MITA, ERIS and MPYS) is a signaling mediator possibly situated downstream of MAVS, and can activate both NF- $\kappa$ B and IRF3 pathways ${ }^{15,41}$. Since TBK1 can activate only the IRF pathway upon

stimulation with cytosolic RNA ${ }^{6,7,15}$, we speculated that ZAPS may function upstream of TBK1. Indeed, the IFNB mRNA expression induced by the exogenous expression of ZAPS in HEK293T cells was abolished by RNAi-mediated downregulation of TBK1 (Supplementary Fig. 3). Notably, RNAi ablation of not only STING or MAVS but also RIG-I in HEK293T cells was found to inhibit the ZAPS-mediated expression of IFNB (Fig. 3f). These results suggest a role for ZAPS as a proximal regulator of RIG-I-mediated signaling. 


\section{ZAPS interacts with RIG-I}

To gain insight into how ZAPS functions as a potentiator of RIG-I activation, we examined whether ZAPS interacts with RIG-I. Confocal analysis of HeLa cells transfected with YFP-tagged ZAPS and Flag-tagged RIG-I revealed that these two proteins are largely colocalized with each other in the cytoplasm $4 \mathrm{~h}$ after 3pRNA stimulation (Fig. 4a). In addition, a fluorescence resonance energy transfer (FRET) signal was detected in the cytoplasmic perinuclear space after 3pRNA stimulation (Fig. 4b). These results indicate that ZAPS directly interacts with RIG-I following ligand stimulation. In an immunoprecipitation assay, the interaction between ZAPS and RIG-I recombinant proteins produced in Escherichia coli was enhanced in the presence of viral RNA (Supplementary Fig. 4a). In HEK293T cells expressing HA-tagged ZAPS and Flag-tagged RIG-I, the interaction between the two proteins was enhanced after stimulation with 3pRNA or viral RNA (Fig. 4c, left panels and Supplementary Fig. 4b), and such ZAPS-RIG-I interaction was similarly observed following infection with NDV (Fig. 4c, middle). We confirmed that endogenous ZAPS protein can associate with endogenous RIG-I in MRC-5 and A549 cells (Fig. 4c, right and Supplementary Fig. 4c). We also determined that the carboxy-terminal region (C-RIG-I; amino acids 218-925) but not the CARDs (N-RIG-I; amino acids 1-284) of RIG-I is required for the interaction with ZAPS (Fig. 4d). In addition, C-RIG-I, but not full-length RIG-I, can preferentially interact with ZAPS even in the absence of 3pRNA stimulation (Fig. 4d), suggesting that the activation-induced conformational change of RIG-I may be necessary for the interaction with ZAPS. However, ZAPS does not bind to either of the helicase domain and/or the C-terminal domain of RIG-I alone (Supplementary Fig. 4d). Both these domains seem to be required for the interaction with ZAPS. On the other hand, full-length ZAPS but not a truncated ZAPS (ZAPS $\triangle \mathrm{zf}$ ) lacking all four zinc fingers that exist in its $\mathrm{N}$-terminal region was found to be co-immunoprecipitated with C-RIG-I (Fig. 4e), suggesting that ZAPS binds to RIG-I via the zinc fingers. In addition, surface plasmon resonance (SPR)-based analysis of recombinant ZAPS and RIG-I showed that ZAPS can interact with RIG-I even in the absence of 3pRNA, with an affinity in the nanomolar range $\left(K_{\mathrm{d}}=116 \mathrm{nM}\right)$ (Supplementary Fig. 4e), but fails to enhance the binding of RIG-I to 3pRNA (data not shown).

We next tested if ZAPS can be involved in the activation of RIG-I. We first examined whether ZAPS is involved in the 3pRNA- or virus-induced RIG-I dimerization or 
oligomerization, which is important in activating the downstream signaling pathway for IFN production ${ }^{42-44}$. The 3pRNA- and NDV-induced formation of RIG-I oligomers was diminished in HEK293T cells with downregulated expression of ZAPS (Fig. 4f and Supplementary Fig. 4f). These data suggest that ZAPS regulates RIG-I oligomerization for the subsequent, robust activation of RIG-I signaling. In vitro biochemical analysis demonstrated that the 3pRNA-induced ATPase activity of purified recombinant RIG-I is much higher in the presence of recombinant ZAPS but not of ZAPS $\triangle$ zf (Fig. 4g). We further found that the association of RIG-I and its adaptor MAVS was significantly attenuated in HEK293T cells treated with siZAPS3 (Fig. 4h). These findings suggest that ZAPS associates with RIG-I to positively modulate RIG-I oligomerization and activation.

\section{Role of ZAPS in RNA virus infection}

To further evaluate the functional role of ZAPS in RIG-I-mediated antiviral responses, we infected A549 cells with influenza virus, which activates the RIG-I-mediated innate signaling $^{10,45}$. RNAi-mediated knockdown of ZAPS expression severely impaired the induction of IFNB and IFNA1 mRNAs (Fig. 5a and Supplementary Fig. 5a) and IFN- $\beta$ protein (Fig. 5b) in response to influenza virus infection $(\mathrm{A} / X-31$ (H3N2) and A/Aichi/2/1968 (H3N2). Induction of IL6, TNF and CXCL10 was also decreased (Fig. 5a). Expression of the viral nucleoprotein ( flu NP) was not affected by ZAPS siRNA knockdown in the early phase of infection (Supplementary Fig. 5b). However, approximately 7-fold higher levels of the viral gene were detected in siZAPS3-treated A549 cells at 72-h post-infection with influenza virus (A/X-31) than in A549 cells treated with control siRNA (Fig. 5c). Moreover, exogenous expression of ZAPS in HEK293T cells resulted in a marked increase in the induction of IFNB following influenza virus infection, accompanied with a notable suppression of viral replication (Fig. 5d). A similar result was also obtained upon infection with NDV, another single-stranded RNA virus (Fig. 5e-g), which is also known to induce type I IFN response via RIG-I (refs. 4,45). Our data suggest that ZAPS exerts its antiviral activity mainly by interactions with the RIG-I-mediated type I IFN pathway (Supplementary Fig. 5c), although an indirect effect on viral replication cannot be completely ruled out.

\section{ZAPS mediates RIG-I activation in human cells}


To further validate the critical role of ZAPS in human cells, we used a ZFN approach ${ }^{46-48}$ for site-specific disruption at the targeted site within exon 3 of the endogenous PARP-13/ZAP gene, ZC3HAV1 (Fig. 6a). Two ZC3HAV1 knockout clones derived from multiploid HEK293T cells were established. Genome sequence analysis showed that clone 32 and clone 89 had four and three distinct ZC3HAV1 gene mutations, respectively (c.1023_1047del25insGTT, c.1027_1046del20, c.1031_1032insATCCA, and c.1033_1034insCAATC for Clone32; c.1035_1036insTCCA, c.1023_1047del25insGTT, and c.1027_1046del20 for Clone89). All these mutations were found to abolish functional protein expression (Fig. 6b). In these two genetically distinct knockout clones NDV infection failed to induce IFNB and other cytokine mRNA expression (Fig. 6c). 3pRNA-induced IFNB expression was similarly inhibited in both clones 32 and 89, and this phenotype was rescued by the exogenous expression of ZAPS (Fig. 6d). These results suggest that ZAPS plays a crucial role in RIG-I-mediated gene induction.

\section{DISCUSSION}

The PARP superfamily plays important roles in multiple biological and pathological processes. Some members of the PARP superfamily are known to directly regulate viral gene expression and replication. However, the role of the PARP superfamily in host immune response mediated by innate sensors remains unknown. In this study we demonstrate that PARP-13 is a strong regulator of RIG-I-mediated antiviral signaling in human cells. We also found that the shorter isoform of PARP-13/ZAP is selectively upregulated by treatment with 3pRNA, possibly through type I IFN signaling. In this respect, it is speculated that ZAPS may play a role as a positive feedback regulator in RIG-I-mediated type I IFN gene induction. Consistently with this scenario, the promoter of ZAP/ZAPS contains ISRE/IRF-E elements. How each isoform is differentially regulated remains unclear.

Activation of RIG-I 3pRNA leads to association with MAVS and activation of the cytosolic protein kinases IKK and TBK1, which in turn activate the transcription factors $\mathrm{NF}-\kappa \mathrm{B}$ and IRF3/7, respectively ${ }^{6,15}$. RIG-I activation after ligand binding is thought to be a multistep process that includes the activation of its ATPase activity, conformational changes and oligomerization ${ }^{42,43}$. Our data demonstrate that ZAPS interacts with RIG-I after 3pRNA stimulation, and potently enhances ATPase activity and oligomerization of RIG-I, possibly by stabilizing the RNA-RIG-I complex, although its underlying 
mechanism needs to be further investigated. Consistently, ZAPS robustly enhances RIG-I-MAVS-mediated downstream gene expression programs, including both type I IFN and proinflammatory cytokine genes. On the other hand, our results also indicate that ZAPS expression alone can slightly enhance IFNB mRNA expression and activation of the IFNB promoter, independently of any ligand or other stimuli. Consistent with these data, ZAPS-RIG-I interaction can be detected before stimulation, albeit very weakly. This disconnect of ligand dependence suggests that ZAPS-RIG-I interaction is enough to promote a low level of signaling but that the overall signaling program is most efficiently triggered by ligand binding to RIG-I in addition to its activation by ZAPS.

Lysine 63 (K63)-linked polyubiquitination of RIG-I, mediated by the RING domain ubiquitin ligase TRIM25, represents another important modification linking RIG-I activation to MAVS ${ }^{17}$. Other mediators of protein modification, such as ISG15, RNF125, CYLD or Riplet/REUL also have a regulatory role in RIG-I activation ${ }^{49-52}$. In addition, unanchored K63-ubiquitin chains, which are not conjugated to any target protein, positively regulate RNA-triggered RIG-I activation through their binding to RIG-I CARDs $^{16}$. Our results support the idea that ZAPS functions as a RIG-I regulator upstream of the ubiquitin-mediated regulatory mechanisms, although further analyses to clarify whether ZAPS may participate in the ubiquitination of RIG-I are required.

AT-rich double-stranded DNA can be transcribed into 5'-triphosphate RNA by DNA-dependent RNA polymerase III, resulting in activation of RIG-I and production of type I interferon and activation of the NF- $\kappa$ B pathway ${ }^{36,37}$. In this context, it can be presumed that ZAPS may also enhance cytosolic DNA-triggered cytokine induction through its positive regulation of RIG-I pathway. This possibility is supported by our preliminary observation that poly(dA:dT)-triggered induction of type I IFNs was significantly abolished in HEK293T cells that had been treated with siRNA targeting ZAPS (S.H., S.S., H.Y. and A.T.; unpublished data).

Our data showing that ZAPS is critically involved in the antiviral innate immune responses against influenza virus and NDV raise the possibility that ZAPS may have an important role in host defence against many other viruses known to activate RIG-I signaling, such as SeV, HCV and $\mathrm{JEV}^{45,53}$. Further investigation on the role of ZAPS in other virally activated PRR-mediated innate signalings is needed. In addition to the important role of ZAPS in RIG-I-mediated response in human cells, we have preliminary 
data, showing the functional role of ZAPS in several murine cells (data not shown), although this remains to be further investigated.

ZAP plays a role in the decay of mRNAs derived from certain viral subsets ${ }^{20,26,39}$. Our current data showing that ZAPS facilitates the activation of innate antiviral mechanisms indicates that this gene might exert a "dual-mode" defence activity against viral infection. These activities might be differentially regulated through the ability to bind two distinct RNA helicases: RIG-I for the activation of host innate immune response and p72 RNA helicase for the degradation of viral RNAs ${ }^{25,26}$. How these activities are regulated remains to be clarified. In addition, due to its dual anti-viral function, ZAPS activation could provide a therapeutic application aimed at enhancing antiviral responses.

\section{ACKNOWLEDGMENTS}

We thank T. Fujita for p-55C1BLuc and p-125Luc, J. Miyazaki for pCAGGS, A. Miyawaki for Venus, H. Kida for NDV, A. Iwai, H. Higashi and J. Hamada for technical help; M. Yamane for purification of human primary CD14 $4^{+}$monocytes; S. Tamura and T. Moriyama for advice on recombinant protein purification. This study was supported in part by Grant-in-Aid for Young Scientists (A) (S.H.), Young Scientists (S) (A.T.), and Scientific Research in Priority Areas (A.T.) from the Ministry of Education, Culture, Sports, Science and Technology of Japan, the IRYOHOJINSHADAN JIKOKAI (H. Tanaka \& N. Takayanagi) (A.T.), the Astellas Foundation for Research on Metabolic Disorders (A.T.), the Kanae Foundation for the Promotion of Medical Science (A.T.), the Kato Memorial Bioscience Foundation (A.T.), and The YASUDA Medical Foundation (A.T.).

\section{AUTHOR CONTRIBUTIONS}

S.H., S.S., H.Y., T.K., C.K., F.K., S.G., S.K., T.Y., M.K., M.S., J.T., M.A. and M.I. planned studies and carried out experiments and analyzed data. D.F. and T.M. contributed to viral infection experiments and helped in data analyses. T.M. and Y.O. performed experiments on fluorescence microscopy and FRET analysis. A.T. supervised the project, designed experiments and wrote the manuscript with comments from the co-authors.

\section{COMPETING FINANCIAL INTERESTS}

The authors declare no competing financial interests. 


\section{Figure legends:}

Figure 1 Involvement of PARP-superfamily members in IFN response to cytosolic nucleic acids. (a) Quantitative RT-PCR (qRT-PCR) analysis of human IFNB mRNA induction levels by stimulation with $1 \mu \mathrm{g} \mathrm{ml}^{-1}$ nucleic acid ligand, namely, 3pRNA, poly(rI:rC) or poly(dA:dT), in HEK293T cells transfected with control or expression vector for each indicated member of the mouse PARP family (upper panel). Error bars in all the panels indicate s.d. $(n=3$, mean + s.d.). Each expression levels of PARP members were analyzed by immunoblotting with anti-HA or anti- $\beta$-actin antibody (lower). NS, nonspecific bands. (b) qRT-PCR of IFNB mRNA following 8 h of 3pRNA stimulation (lower left) and luciferase activity of a p-125Luc reporter plasmid measured $48 \mathrm{~h}$ after transfection (lower right) of HEK293T cells with increasing doses of control (C) or HA-tagged short or long isoform (ZAPS or ZAP) expression vector. The expressions of both isoforms were confirmed to be at almost the same levels by immunoblotting (upper panel). (c) qRT-PCR of ZAPS and ZAP mRNAs in HEK293T stimulated with 3pRNA (left panels) or IFN- $\alpha$ (500 $\mathrm{U} \mathrm{ml}^{-1}$; right panels) for the time frame indicated. Values are presented as fold changes relative to the data at $0 \mathrm{~h}$. (d) qRT-PCR of ZAPS and ZAP mRNAs by 3pRNA in human primary CD14 ${ }^{+}$monocytes purified from PBMCs. Values are presented as fold changes relative to the data at 0 h. $* P<0.05, * * P<0.01$. Data are representative of two or three experiments. RE, relative expression units.

Figure 2 ZAPS is a potent stimulator of RIG-I-mediated type I IFN response activated by 3pRNA. (a) qRT-PCR analysis of IFNA1, IFNA4 and IFNB mRNAs in HEK293T cells transfected with control (white bar) or ZAPS (black) expression vector for the indicated time frame following 3pRNA stimulation. (b,c) qRT-PCR of IFNB mRNA 
following $8 \mathrm{~h}$ of 3pRNA stimulation (b) and luciferase activity of a p-125Luc reporter plasmid following $16 \mathrm{~h}$ of 3pRNA stimulation (c) in HEK293T cells transfected with increasing doses of control (C) or ZAPS expression vector. (d) qRT-PCR of IFNB mRNA following $8 \mathrm{~h}$ of 3pRNA stimulation (left panel) and ELISA of IFN- $\beta$ measured 24 h post-stimulation (right) in HEK293T cells transfected with control (siControl) or each of three different siRNAs targeting ZAPS (siZAPS1, 2 and 3). ND, not detected. (e) qRT-PCR of IFNB mRNA following $8 \mathrm{~h}$ of 3pRNA stimulation in HEK293T cells co-transfected with control, mouse ZAPS (mZAPS) or PARP-1 expression vector alone with siZAPS3. (f) qRT-PCR of IFNA1 and IFNB mRNAs following 8h of stimulation with flu vRNA in A549 cells transfected with siControl or siZAPS3. (g,h) qRT-PCR of type I IFN mRNAs following $8 \mathrm{~h}$ of 3pRNA stimulation in human primary CD14 ${ }^{+}$ monocytes (g) and fibroblasts (MRC-5) (h) treated with siControl or siZAPS3 for $48 \mathrm{~h}$. ${ }^{*} P<0.05,{ }^{* *} P<0.01$. Data are representative of two or three experiments.

Figure 3 ZAPS activates both NF- $\kappa$ B and IRF3 transcriptional pathways in a RIG-I- and MAVS-dependent manner. (a) qRT-PCR analysis of TNF, IL6 and CXCL10 mRNAs following 8h of 3pRNA stimulation in A549 cells transfected with siControl or siZAPS3. (b) Luciferase activity of a pNF-кB-Luc reporter plasmid in HEK293T cells, assessed as described in Figure 2c. (c) Luciferase activity of a pNF-кB-Luc reporter plasmid in TLR8-expressing HEK293T cells, measured $16 \mathrm{~h}$ after 3pRNA or R848 stimulation. (d) Native PAGE and immunoblotting for the detection of 3pRNA-induced dimerization of endogenous IRF3 in HEK293T cells treated with siControl or siZAPS3 (upper panel). Lysates from HEK293T cells transfected with control or MAVS expression vector were used as a negative or positive control, respectively. The relative band intensities of IRF3 dimer quantified using a densitometer were normalized to those of IRF3 monomer (bottom). (e) EMSA of NF-кB activation induced by 3pRNA stimulation in HEK293T 
cells treated with siControl or siZAPS3. (f) qRT-PCR of IFNB mRNA following $48 \mathrm{~h}$ of the co-transfection of HEK293T cells with siControl, siSTING, siMAVS or siRIG-I along with ZAPS expression vector. ${ }^{*} P<0.05$, ${ }^{* *} P<0.01$. Data are representative of two or three experiments.

Figure 4 ZAPS interacts with RIG-I to positively modulate the RIG-I activity. (a) Fluorescence confocal images of HeLa cells co-transfected with YFP-ZAPS and Flag-RIG-I (secondarily visualized with Alexa Fluor 594; Alexa Fluor 594-RIG-I), following $4 \mathrm{~h}$ of 3pRNA stimulation. The graph shows a linescan of fluorescence intensity from a white line drawn in the right panel (magnification of area outlined in the main image). (b) Intermolecular FRET of the interaction between YFP-ZAPS and CFP-RIG-I following 3pRNA stimulation in HeLa cells. FRET ${ }^{\mathrm{C}}$ CFP values were plotted in the graph (Bars, mean values). Representative fluorescence images of FRET ${ }^{\mathrm{c}}$ (corrected FRET; displayed in pseudo-color mode) are shown (bottom). (c) Co-immunoprecipitation of HA-ZAPS with Flag-RIG-I in HEK293T cells after treatment with 3pRNA (left panel) or NDV (middle). Cell lysates were analyzed by immunoprecipitation with anti-Flag antibody, followed by immunoblotting with anti-HA or anti-Flag antibody. Immunoblot with anti-HA antibody for whole cell lysates (WCL) is also shown (bottom). Co-immunoprecipitation of endogenous proteins of ZAPS and RIG-I in MRC-5 cells following 6 h of 3pRNA stimulation was shown (right). (d) Interaction of HA-ZAPS with Flag-RIG-I or its deletion mutants in HEK293T cells following $6 \mathrm{~h}$ of 3pRNA stimulation was assessed as described in c. (e) Interaction of Flag-C-RIG-I with HA-ZAPS or its mutants (N1-ZAPS, N2-ZAPS, and ZAPS $\triangle$ zf; right panel) in HEK293T cells was analyzed as in c. NS, nonspecific bands. (f) Native PAGE and immunoblotting for the detection of Flag-RIG-I oligomerization induced by NDV infection (16 h) in siZAPS3-treated HEK293T cells. The relative band intensities of RIG-I oligomer were normalized to those of RIG-I monomer (bottom). (g) ATPase 
activity of recombinant RIG-I protein was measured after the addition of 3pRNA in the presence of increasing doses of recombinant GST-ZAPS or ZAPS $\Delta \mathrm{zf}$. $* * P<0.01$. (h) Association of Flag-RIG-I with HA-MAVS following $6 \mathrm{~h}$ of 3pRNA stimulation in siZAPS3- or siControl-treated HEK293T cells was assessed as in c. Data are representative of two or three experiments.

Figure 5 ZAPS is a key regulator in RIG-I-mediated induction of type I IFNs and antiviral innate defence. (a-c) qRT-PCR analysis of expression levels of IFNB, IFNA1, IL6, TNF and CXCL10 mRNAs, nucleoprotein (flu NP) gene (a,c) and ELISA of IFN- $\beta$ protein (b) in siControl- or siZAPS3-treated A549 cells, following infection with influenza virus $(\mathrm{A} / X-31$; multiplicity of infection, MOI, 1.0) for the indicated time periods. (d) qRT-PCR of IFNB mRNA induced by infection with influenza virus (MOI 1.0) in HEK293T cells transfected with control or ZAPS expression vector (left). Virus titers (PFU; plaque-forming unit) were determined by plaque-forming assay after $48 \mathrm{~h}$ of infection (right). (e-g) qRT-PCR of expression levels of IFNB and IFNA1 mRNAs, NDV $F$ gene $(\mathbf{e , g})$ and ELISA of IFN- $\beta$ protein (f) in siControl- or siZAPS3-treated A549 cells, following infection with NDV for the indicated periods. $* P<0.05$, $* * P<0.01$. Data are representative of two or three experiments.

Figure 6 Crucial role of ZAPS in cytokine gene induction by NDV infection. (a) Strategy of site-specific gene disruption for generation of human ZC3HAV1 knockout cells. Schematic representation of the ZFN-targeting site within the exon 3 of human ZC3HAV1. ZFN-L and ZFN-R are recognition sites of the designed ZFN pairs. (b) ZAPS 
protein expression in each knockout clone and parental HEK293T cells (WT) was assessed by immunoprecipitation/immunoblotting with anti-human ZCCHV antibody. (c) qRT-PCR analysis of induction levels of IFNB, TNF and CXCL10 mRNAs in response to NDV infection (8 h) in HEK293T cells (WT) or ZC3HAV1 knockout clones. (d) qRT-PCR of IFNB mRNA following $8 \mathrm{~h}$ of 3pRNA stimulation in parental HEK293T cells (WT) and each knockout clone transfected with control (C) or ZAPS (Z) expression vector. $* P<0.05$, $* * P<0.01$. Data are representative of two or three experiments.

\section{METHODS}

Methods any associated references are available in the online version of the paper at http://www.nature.com/natureimmunology/.

\section{ONLINE METHODS}

Cells, antibodies and reagents. HEK293T, A549, and HeLa cells were obtained from ATCC. MRC-5 (RCB0218) cells were provided by the RIKEN BRC through the National Bio-Resource Project of the MEXT, Japan. All cells were cultured as previously described $^{54}$. Peripheral blood mononuclear cells (PBMC) were isolated from peripheral venous blood of healthy volunteers (provided by Hokkaido Red Cross Blood Center) by density gradient centrifugation. Human primary CD14 ${ }^{+}$monocytes $\left(>95 \% \mathrm{CD}^{+} 4^{+}\right.$as determined by flow cytometric analysis) were obtained from PBMC, by magnetic cell sorting (MACS) using magnetic microbeads according to the manufacturer's instructions (Miltenyi Biotec). Experiments involving human blood were approved by the institutional review board. Antibodies against the following proteins were purchased as indicated: Flag (M2; Sigma), HA (5D8; MBL), GFP (632375; Clontech), RIG-I (8048849; ENZO life science), IRF3 (IRF35I218-2; MBR), ZCCHV (ab73369; Abcam), 
IFNAR-2 (MMHAR-2; PBL) and $\beta$-actin (AC-15; Sigma). 3pRNA was prepared as previously reported $^{55}$. Poly(dA:dT), poly(rI:rC), R848, and human IFN- $\alpha$ were purchased from Sigma, GE Healthcare, Alexis, and Pepro Tech, respectively. Transfection with nucleic acid ligands into the cytoplasm was performed with Lipofectamine 2000 (Invitrogen).

Plasmids and gene transfer. Complementary DNAs encoding mouse Parp-1, Parp-2, Parp-7/Tiparp, Parp-9, Parp-12, Parp-13/Zc3hav1, human ZC3HAV1 isoform 1 (ZAP) and 2 (ZAPS), MAVS, RIG-I and the related mutants of ZAPS and RIG-I, were obtained by polymerase chain reaction with reverse transcription (RT-PCR) on total RNAs from mouse embryonic fibroblasts or HEK293T cells, and then cloned into the TArget Clone -Plus- (TOYOBO). To generate YFP-, Flag- and HA-tagged proteins, the cDNAs were cloned into the XhoI and NotI sites of the pCAGGS-YFP, pCXN2-CFP, pCXN2-Flag and pIRM-3HA vectors. TLR8 expression vector was obtained from Invivogen. The nucleotide sequences for cDNAs were confirmed with BigDye terminator v3.1 sequencing Kit (Applied Biosystems). The pCAGGS and Venus, which we refer to as YFP, were provided by J. Miyazaki and A. Miyawaki, respectively. To construct expression plasmids for recombinant proteins, ZAPS, ZAPS $\Delta \mathrm{zf}$ and RIG-I, the former two cDNAs were cloned into the EcoRI and NotI sites of the pGEX-6P-1 (GE Healthcare), and the latter one into the SalI and NotI sites of pGEX-4T-3 (GE Healthcare). FuGENE6 (Roche) reagent was used for gene transfer with lipid transfection.

Quantitative RT-PCR analysis. Total RNA was subjected to qRT-PCR by using ReverTra Ace (TOYOBO) and SYBR Premix Ex Taq (TAKARA), and analyzed on a StepOnePlus (Applied Biosystems). Data were normalized by the expression level of $A C T B$ or GAPDH in each sample. Sequence information for PCR primers is shown in

\section{Supplementary Table 1.}


RNA interference. Chemically synthesized 21-nucleotide siRNAs including Control siRNA (siPerfect negative control) were obtained from Sigma. siRNA sequences are listed in Supplementary Table 2. At $48 \mathrm{~h}$ after transfection with $50 \mathrm{nM}$ siRNA in $2.0 \mu \mathrm{l}$ of Lipofectamine 2000 or Lipofectamine RNAiMAX (Invitrogen), cells were used for further experiments.

Reporter analysis. HEK293T cells seeded on 24-well plates were transiently co-transfected with $100 \mathrm{ng}$ of each luciferase reporter plasmid, pNF-кB-Luc (Clontech), p-55C1BLuc and p-125Luc (provided by T. Fujita) together with the increasing doses of expression vector or control vector. As an internal control, 10 ng of Renilla luciferase reporter plasmid was transfected simultaneously. Forty-eight hours after transfection, cells were stimulated by 3pRNA or R848, and luciferase activity was measured with Dual-luciferase reporter assay system (Promega).

Immunoblotting/immnoprecipitation assay and ELISA. Cell lysis, immunoblotting and immunoprecipitation assay were conducted as described previously ${ }^{54}$. Dimerization/oligomerization of IRF3 or RIG-I was assessed by native PAGE, followed by immunoblot assay, as described previously ${ }^{13,42,54}$. IFN- $\beta$ protein levels in culture supernatants were measured by ELISA following the manufacture's protocol (PBL).

Electrophoretic mobility shift assay (EMSA). Equal amounts (40 $\mu \mathrm{g})$ of nuclear protein extract were incubated with ${ }^{32} \mathrm{P}$-radiolabelled oligonucleotide probe containing a consensus NF- $\kappa \mathrm{B}$ binding sequence, and the subsequent assay was carried out as described previously ${ }^{54}$. 
Fluorescence analysis. HeLa cells were transfected with $1.0 \mu \mathrm{g}$ per well YFP-ZAPS and Flag-RIG-I expression vectors. Flag-RIG-I was stained with anti-Flag antibody and the appropriate secondary antibody conjugated with Alexa Fluor 594 (Molecular probes). Localization of RIG-I and ZAPS was visualized on an OLYMPAS BX61 confocal microscope. FRET analysis was performed as described previously ${ }^{54}$. The filters used in this study include U-MCFPHQ excitation/emission filters (OLYMPAS) for the YFP and CFP image and CFP/YFP-2X2M-A for a FRET (Semrock). As a dichroic mirror, a UMREF glass reflector (Semrock) was used. Corrected FRET (FRET ${ }^{\mathrm{C}}$ ) was calculated using the equation: FRET $^{\mathrm{C}}=$ FRET $-0.5835 \times$ CFP $-0.1139 \times$ YFP, YFP and CFP channels, respectively.

Purification of recombinant proteins and in vitro ATPase assay. GST-tagged ZAPS, ZAPS $\Delta \mathrm{zf}$ and RIG-I proteins were expressed in E. coli BL21 and purified with glutathione-Sepharose 4B beads (GE Healthcare), according to the manufacturer's instruction. ATPase activities were measured using a phosphate release assay using the Biomol green phosphate assay reagent (BIOMOL). In an ATPase reaction buffer (20 mM Tris-HCl, pH 8.0, $1.5 \mathrm{mM} \mathrm{MgCl}_{2}$ and 1.5 mM DTT), $0.5 \mu \mathrm{g}$ of purified RIG-I protein and the indicated amounts of ZAPS or ZAPS $\Delta$ zf protein were incubated with $1 \mu \mathrm{g}$ of 3pRNA for $15 \mathrm{~min}$ at $37^{\circ} \mathrm{C}$. The mixture was further incubated with $5 \mathrm{nmol}$ ATP at $37^{\circ} \mathrm{C}$ for 15 min. Phosphate levels were then determined according to the manufacturer's protocol.

Viral infection and plaque-forming assay. Cells were infected with 1.0 or 0.1 MOI of influenza A viruses (A/X-31 (H3N2)) for $1 \mathrm{~h}$ in an infection-medium (serum-free MEM containing amino acids and trypsin) at $37^{\circ} \mathrm{C}$. Plaque forming assay was performed by using MDCK cells. A549 and HEK293T cells were infected with a concentration of 25 hemagglutinating units (HAU) of NDV (provided by H.Kida) per $1 \times 10^{6}$ cells, as described previously ${ }^{54}$. 
Establishment of human ZC3HAV1 knockout cells. ZC3HAV1 knockout cells were generated by utilizing CompoZr ${ }^{\mathrm{TM}}$ knockout ZFN Technology (Sigma) according to the manufacturer's instruction ${ }^{46}$. Briefly, mRNAs or vectors coding for those ZFN proteins were transfected into HEK293T cells by using Lipofectamine 2000. Three days after transfection, cells were replated into 96-well plates by the limiting dilution ( 0.5 cell per well). The resulting genetic alterations were examined by PCR/sequencing and immunoprecipitation/immunoblotting.

Statistical analysis. Differences between control and experimental groups were evaluated using the Student's $t$-test.

\section{References}

1. Takeuchi, O. \& Akira, S. Pattern recognition receptors and inflammation. Cell 140, 805-820 (2010).

2. Takaoka, A. \& Taniguchi, T. Cytosolic DNA recognition for triggering innate immune responses. Adv. Drug Deliv. Rev. 60, 847-857 (2008).

3. Katze, M., Fornek, J., Palermo, R., Walters, K. \& Korth, M. Innate immune modulation by RNA viruses: emerging insights from functional genomics. Nat. Rev. Immunol. 8, 644-654 (2008).

4. Yoneyama, M. et al. The RNA helicase RIG-I has an essential function in double-stranded RNA-induced innate antiviral responses. Nat. Immunol. 5, 730-737 (2004).

5. Yoneyama, M. et al. Shared and unique functions of the DExD/H-box helicases RIG-I, MDA5, and LGP2 in antiviral innate immunity. J. Immunol. 175, 2851-2858 (2005). 
6. Yoneyama, M. \& Fujita, T. RNA recognition and signal transduction by RIG-I-like receptors. Immunol. Rev. 227, 54-65 (2009).

7. Takeuchi, O. \& Akira, S. Innate immunity to virus infection. Immunol. Rev. 227, 75-86 (2009).

8. Rehwinkel, J. \& Reis e Sousa, C. RIGorous detection: exposing virus through RNA sensing. Science 327, 284-286 (2010).

9. Hornung, V. et al. 5'-Triphosphate RNA is the ligand for RIG-I. Science 314, 994-997 (2006).

10. Pichlmair, A. et al. RIG-I-mediated antiviral responses to single-stranded RNA bearing 5'-phosphates. Science 314, 997-1001 (2006).

11. Kawai, T. et al. IPS-1, an adaptor triggering RIG-I- and Mda5-mediated type I interferon induction. Nat. Immunol. 6, 981-988 (2005).

12. Meylan, E. et al. Cardif is an adaptor protein in the RIG-I antiviral pathway and is targeted by hepatitis C virus. Nature 437, 1167-1172 (2005).

13. Seth, R.B., Sun, L., Ea, C.K. \& Chen, Z.J. Identification and characterization of MAVS, a mitochondrial antiviral signaling protein that activates NF-kappaB and IRF 3. Cell 122, 669-682 (2005).

14. $\mathrm{Xu}, \mathrm{L}$. et al. VISA is an adapter protein required for virus-triggered IFN-beta signaling. Mol. Cell 19, 727-740 (2005).

15. Nakhaei, P., Genin, P., Civas, A. \& Hiscott, J. RIG-I-like receptors: sensing and responding to RNA virus infection. Semin. Immunol. 21, 215-222 (2009).

16. Zeng, W. et al. Reconstitution of the RIG-I pathway reveals a signaling role of unanchored polyubiquitin chains in innate immunity. Cell 141, 315-330 (2010).

17. Gack, M. et al. TRIM25 RING-finger E3 ubiquitin ligase is essential for RIG-I-mediated antiviral activity. Nature 446, 916-920 (2007).

18. Cui, J. et al. NLRC5 negatively regulates the NF-kappaB and type I interferon signaling pathways. Cell 141, 483-496 (2010).

19. Hakme, A., Wong, H.K., Dantzer, F. \& Schreiber, V. The expanding field of poly(ADP-ribosyl)ation reactions. 'Protein Modifications: Beyond the Usual Suspects' Review Series. EMBO Rep. 9, 1094-1100 (2008).

20. Gao, G., Guo, X. \& Goff, S.P. Inhibition of retroviral RNA production by ZAP, a CCCH-type zinc finger protein. Science 297, 1703-1706 (2002). 
21. Hassa, P.O. \& Hottiger, M.O. The diverse biological roles of mammalian PARPS, a small but powerful family of poly-ADP-ribose polymerases. Front. Biosci. 13, 3046-3082 (2008).

22. Schreiber, V., Dantzer, F., Ame, J.C. \& de Murcia, G. Poly(ADP-ribose): novel functions for an old molecule. Nat. Rev. Mol. Cell Biol. 7, 517-528 (2006).

23. Tempera, I. et al. Regulation of Epstein-Barr virus OriP replication by poly(ADP-ribose) polymerase 1. J. Virol. 84, 4988-4997 (2010).

24. Ohsaki, E. et al. Poly(ADP-ribose) polymerase 1 binds to Kaposi's sarcoma-associated herpesvirus (KSHV) terminal repeat sequence and modulates KSHV replication in latency. J. Virol. 78, 9936-9946 (2004).

25. Chen, G., Guo, X., Lv, F., Xu, Y. \& Gao, G. p72 DEAD box RNA helicase is required for optimal function of the zinc-finger antiviral protein. Proc. Natl. Acad. Sci. U. S. A. 105, 4352-4357 (2008).

26. Zhu, Y. \& Gao, G. ZAP-mediated mRNA degradation. RNA Biol. 5, 65-67 (2008).

27. Hassa, P., Buerki, C., Lombardi, C., Imhof, R. \& Hottiger, M. Transcriptional coactivation of nuclear factor-kappaB-dependent gene expression by p300 is regulated by poly(ADP)-ribose polymerase-1. J. Biol. Chem. 278, 45145-45153 (2003).

28. Oliver, F. et al. Resistance to endotoxic shock as a consequence of defective NF-kappaB activation in poly (ADP-ribose) polymerase-1 deficient mice. EMBO J. 18, 4446-4454 (1999).

29. Aguiar, R., Takeyama, K., He, C., Kreinbrink, K. \& Shipp, M. B-aggressive lymphoma family proteins have unique domains that modulate transcription and exhibit poly(ADP-ribose) polymerase activity. J. Biol. Chem. 280, 33756-33765 (2005).

30. Juszczynski, P. et al. BAL1 and BBAP are regulated by a gamma interferon-responsive bidirectional promoter and are overexpressed in diffuse large B-cell lymphomas with a prominent inflammatory infiltrate. Mol. Cell Biol. 26, 5348-5359 (2006).

31. Yelamos, J. et al. PARP-2 deficiency affects the survival of CD4+CD8+ double-positive thymocytes. EMBO J. 25, 4350-4360 (2006). 
32. Kofler, J. et al. Differential effect of PARP-2 deletion on brain injury after focal and global cerebral ischemia. J. Cereb. Blood Flow Metab. 26, 135-141 (2006).

33. Ma, Q., Baldwin, K.T., Renzelli, A.J., McDaniel, A. \& Dong, L. TCDD-inducible poly(ADP-ribose) polymerase: a novel response to 2,3,7,8-tetrachlorodibenzo-p-dioxin. Biochem. Biophys. Res. Commun. 289, 499-506 (2001).

34. Kutsch, S., Degrandi, D. \& Pfeffer, K. Immediate lymphotoxin beta receptor-mediated transcriptional response in host defense against $\mathrm{L}$. monocytogenes. Immunobiology 213, 353-366 (2008).

35. Kato, H. et al. Length-dependent recognition of double-stranded ribonucleic acids by retinoic acid-inducible gene-I and melanoma differentiation-associated gene 5 . J. Exp. Med. 205, 1601-1610 (2008).

36. Chiu, Y.H., Macmillan, J.B. \& Chen, Z.J. RNA polymerase III detects cytosolic DNA and induces type I interferons through the RIG-I pathway. Cell 138, 576-591 (2009).

37. Ablasser, A. et al. RIG-I-dependent sensing of poly(dA:dT) through the induction of an RNA polymerase III-transcribed RNA intermediate. Nat. Immunol. 10, 1065-1072 (2009).

38. Kerns, J.A., Emerman, M. \& Malik, H.S. Positive selection and increased antiviral activity associated with the PARP-containing isoform of human zinc-finger antiviral protein. PLoS Genet. 4, e21 (2008).

39. Guo, X., Carroll, J.W., Macdonald, M.R., Goff, S.P. \& Gao, G. The zinc finger antiviral protein directly binds to specific viral mRNAs through the CCCH zinc finger motifs. J. Virol. 78, 12781-12787 (2004).

40. Wang, N. et al. Viral induction of the zinc finger antiviral protein is IRF3-dependent but NF-kappaB-independent. J. Biol. Chem. 285, 6080-6090 (2010).

41. Bowzard, J.B., Ranjan, P., Sambhara, S. \& Fujita, T. Antiviral defense: RIG-Ing the immune system to STING. Cytokine Growth Factor Rev. 20, 1-5 (2009).

42. Saito, T. et al. Regulation of innate antiviral defenses through a shared repressor domain in RIG-I and LGP2. Proc. Natl. Acad. Sci. U. S. A. 104, 582-587 (2007).

43. Cui, S. et al. The C-terminal regulatory domain is the RNA 5'-triphosphate sensor of RIG-I. Mol. Cell 29, 169-179 (2008). 
44. Schmidt, A. et al. 5'-triphosphate RNA requires base-paired structures to activate antiviral signaling via RIG-I. Proc. Natl. Acad. Sci. U. S. A. 106, 12067-12072 (2009).

45. Kato, H. et al. Differential roles of MDA5 and RIG-I helicases in the recognition of RNA viruses. Nature 441, 101-105 (2006).

46. Urnov, F. et al. Highly efficient endogenous human gene correction using designed zinc-finger nucleases. Nature 435, 646-651 (2005).

47. Foley, J. et al. Targeted mutagenesis in zebrafish using customized zinc-finger nucleases. Nat. Protoc. 4, 1855-1867 (2009).

48. Lee, H., Kim, E. \& Kim, J. Targeted chromosomal deletions in human cells using zinc finger nucleases. Genome Res. 20, 81-89 (2010).

49. Yuan, W. \& Krug, R. Influenza B virus NS1 protein inhibits conjugation of the interferon (IFN)-induced ubiquitin-like ISG15 protein. EMBO J. 20, 362-371 (2001).

50. Arimoto, K. et al. Negative regulation of the RIG-I signaling by the ubiquitin ligase RNF125. Proc. Natl. Acad. Sci. U. S. A. 104, 7500-7505 (2007).

51. Friedman, C. et al. The tumour suppressor CYLD is a negative regulator of RIG-I-mediated antiviral response. EMBO Rep. 9, 930-936 (2008).

52. Oshiumi, H., Matsumoto, M., Hatakeyama, S. \& Seya, T. Riplet/RNF135, a RING finger protein, ubiquitinates RIG-I to promote interferon-beta induction during the early phase of viral infection. J. Biol. Chem. 284, 807-817 (2009).

53. Saito, T., Owen, D.M., Jiang, F., Marcotrigiano, J. \& Gale, M., Jr. Innate immunity induced by composition-dependent RIG-I recognition of hepatitis C virus RNA. Nature 454, 523-527 (2008).

54. Takaoka, A. et al. DAI (DLM-1/ZBP1) is a cytosolic DNA sensor and an activator of innate immune response. Nature 448, 501-505 (2007).

55. Takahasi, K. et al. Solution structures of cytosolic RNA sensor MDA5 and LGP2 C-terminal domains: identification of the RNA recognition loop in RIG-I-like receptors. J. Biol. Chem. 284, 17465-17474 (2009). 
a

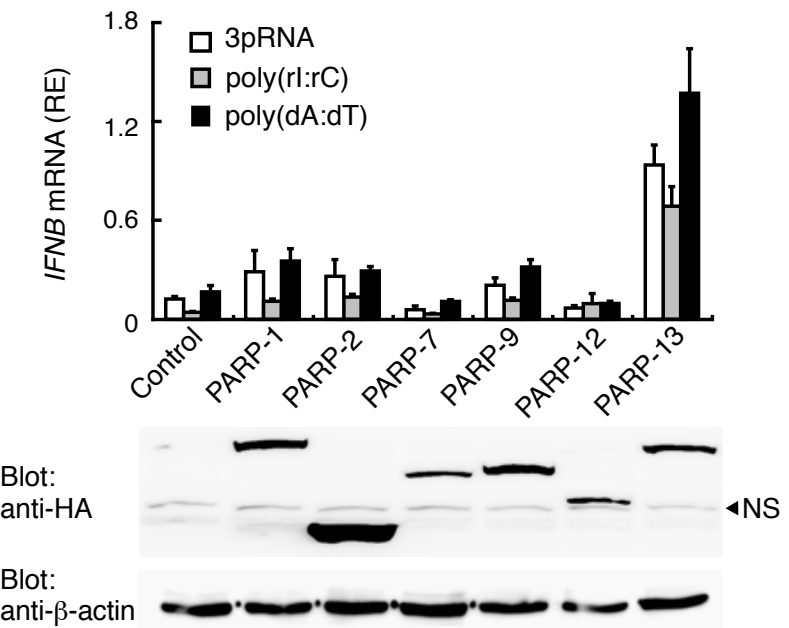

b

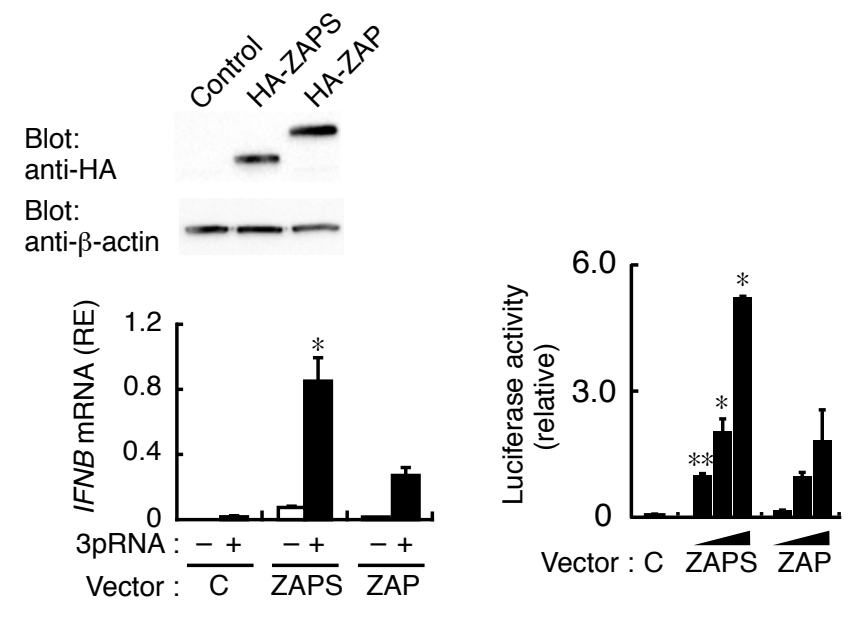

C
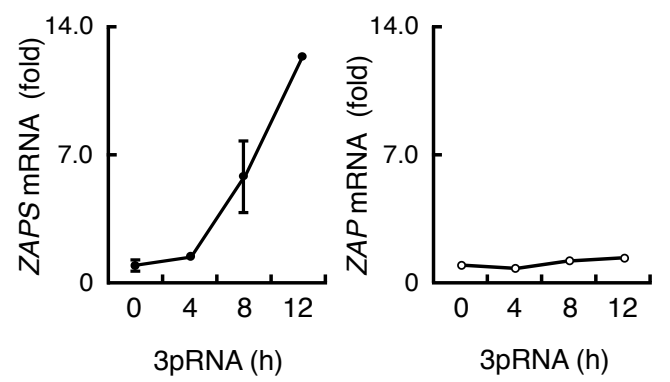

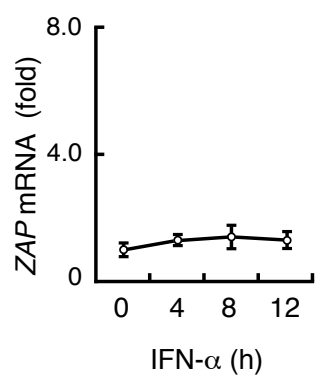

d

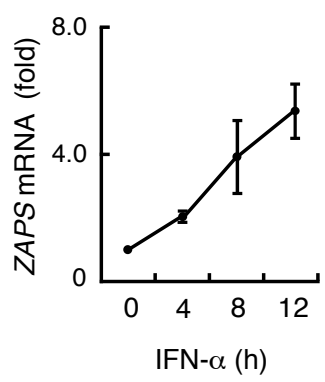

IFN- $\alpha$ (h)
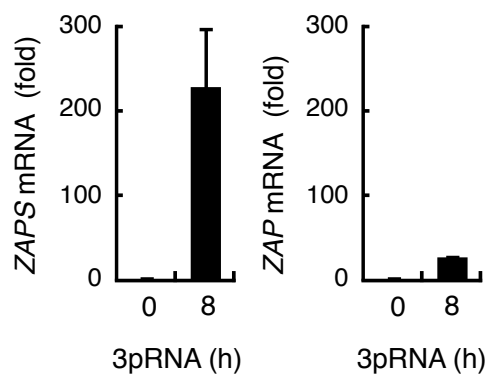

Fig. 1 
a

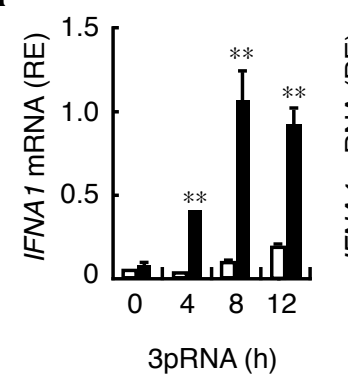

d

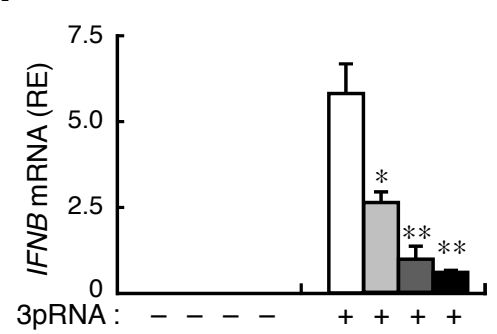

खे $\omega^{\prime} s^{2} s^{3} \mathrm{O}^{1} s^{2} s^{3}$

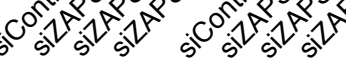

b

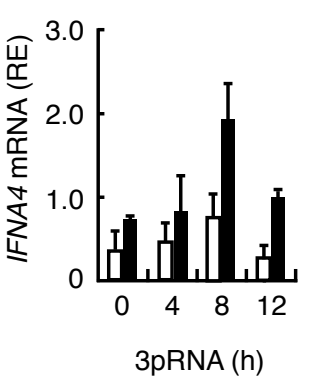

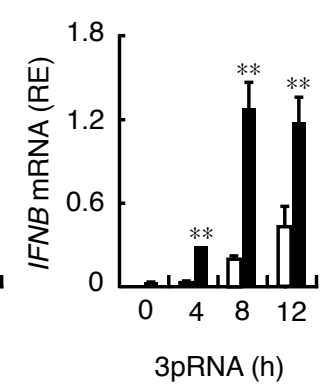

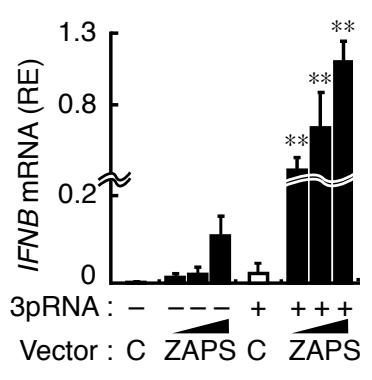

C

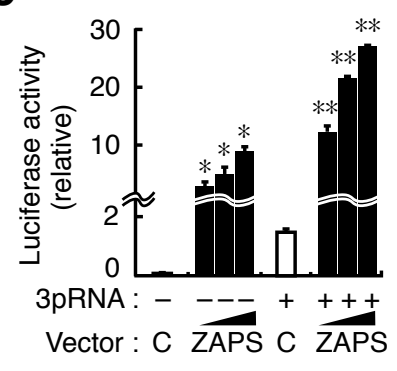

e
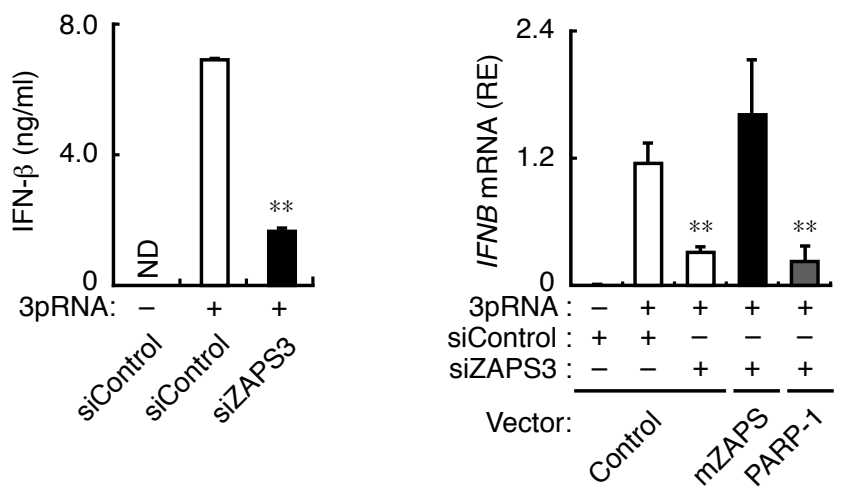

f
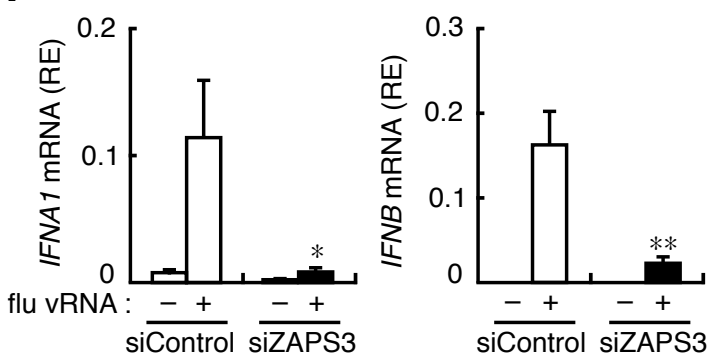

g

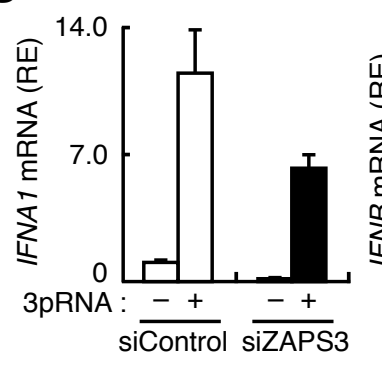

h

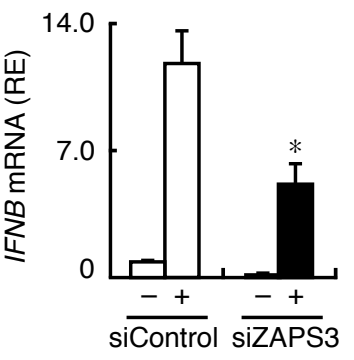

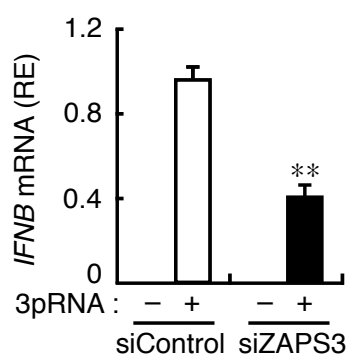

Fig. 2 
a
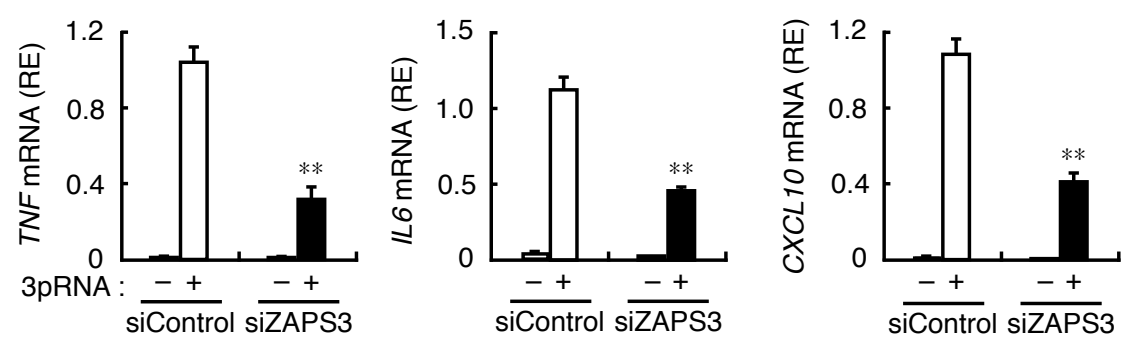

b

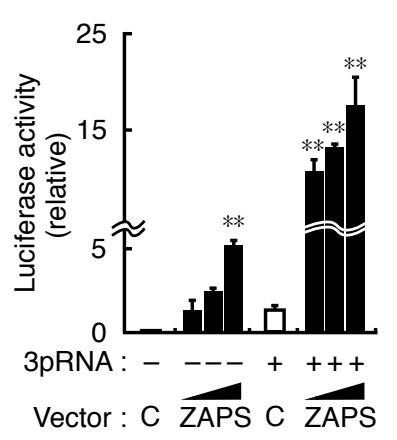

C

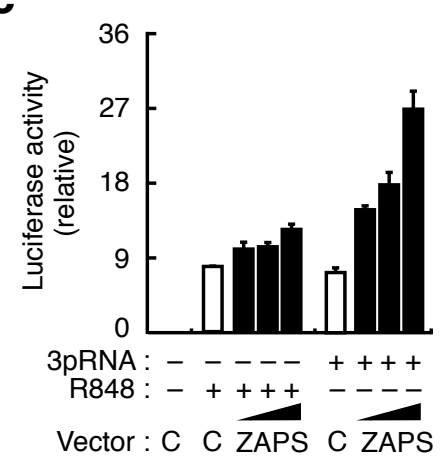

d Blot: anti-IRF3
3pRNA $(\mu \mathrm{g} / \mathrm{ml}):$
0

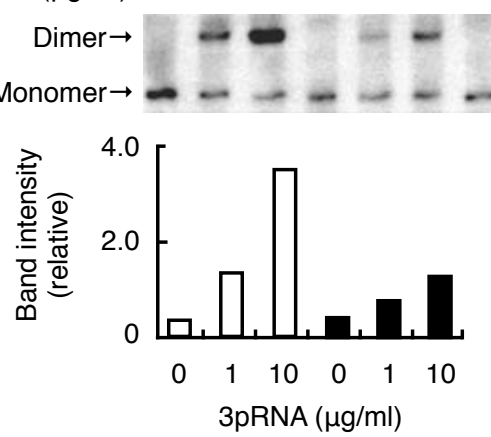

e

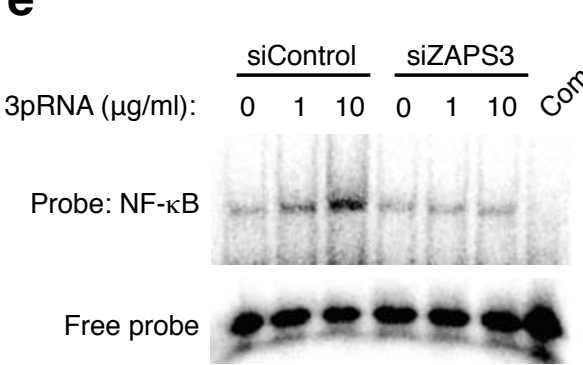

f

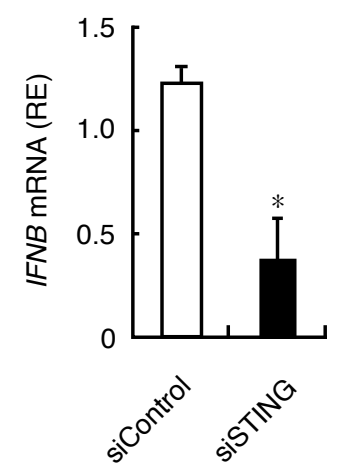

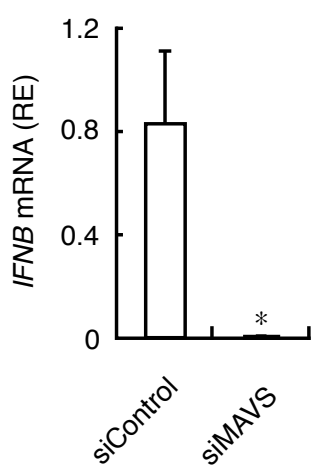

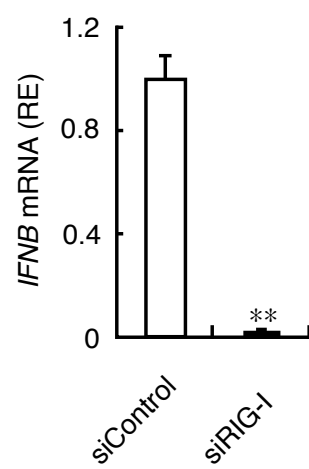

Fig. 3 
a

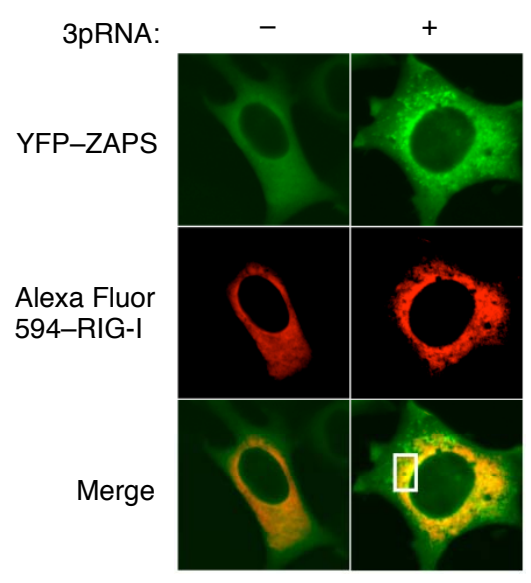

b

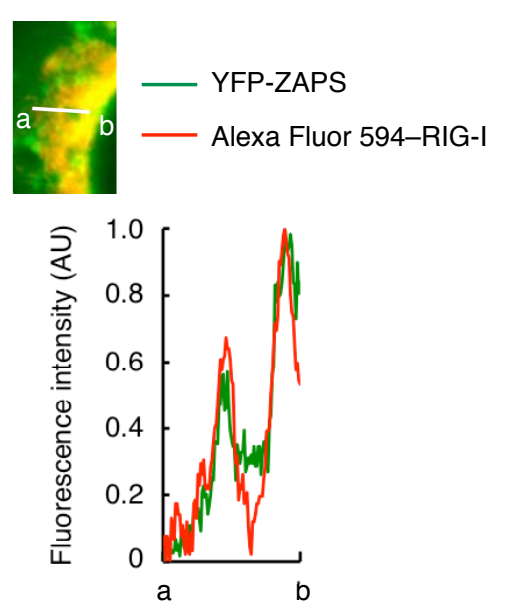

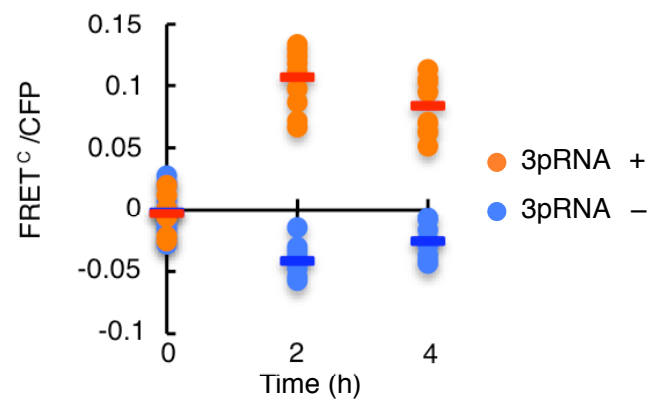

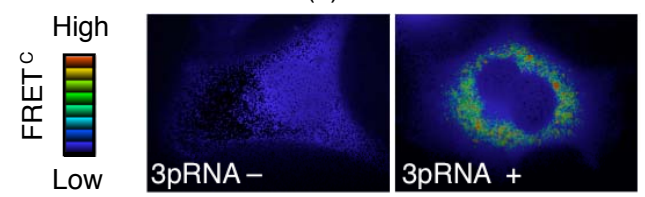

C

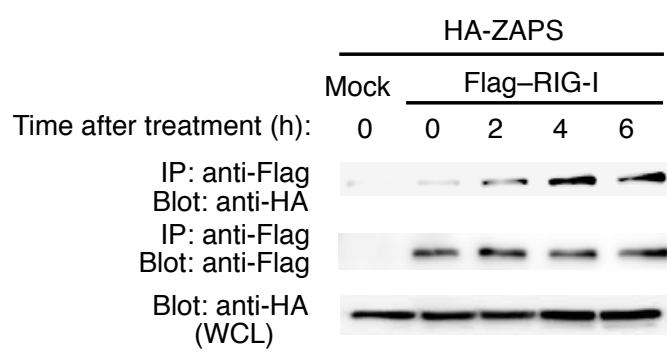

d

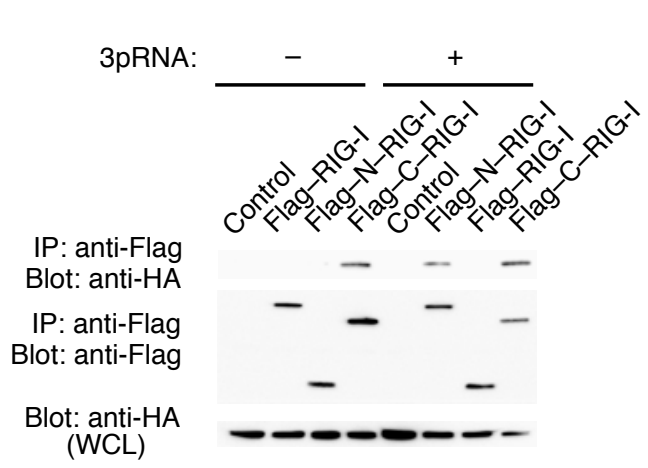

f siControl siZAPS3
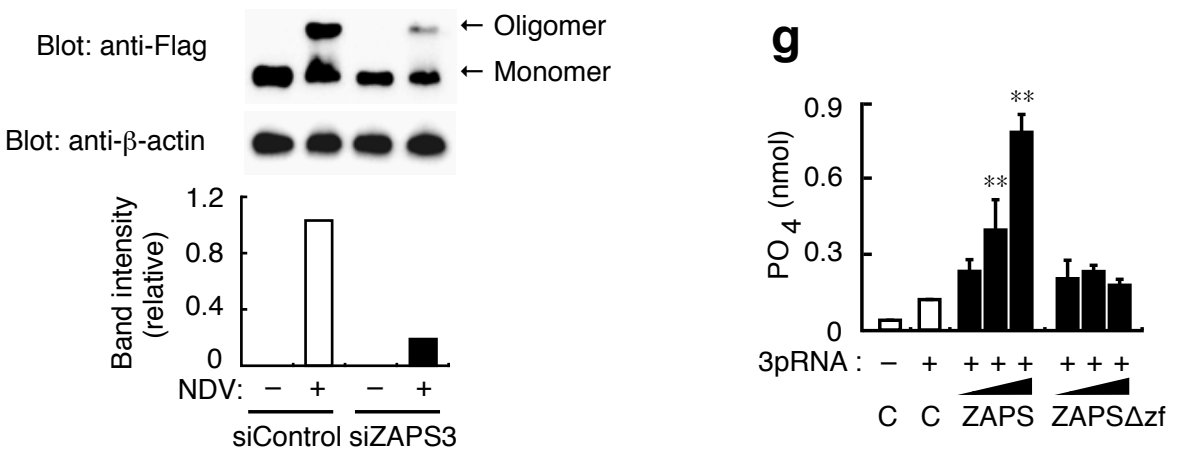

h

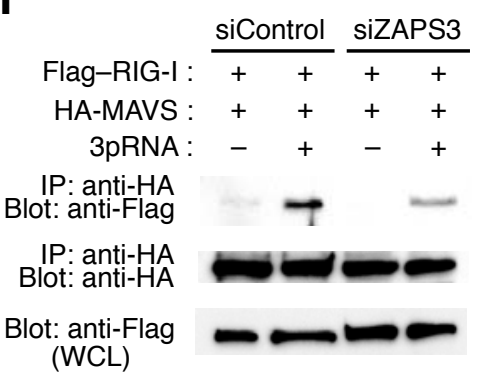

Fig. 4 

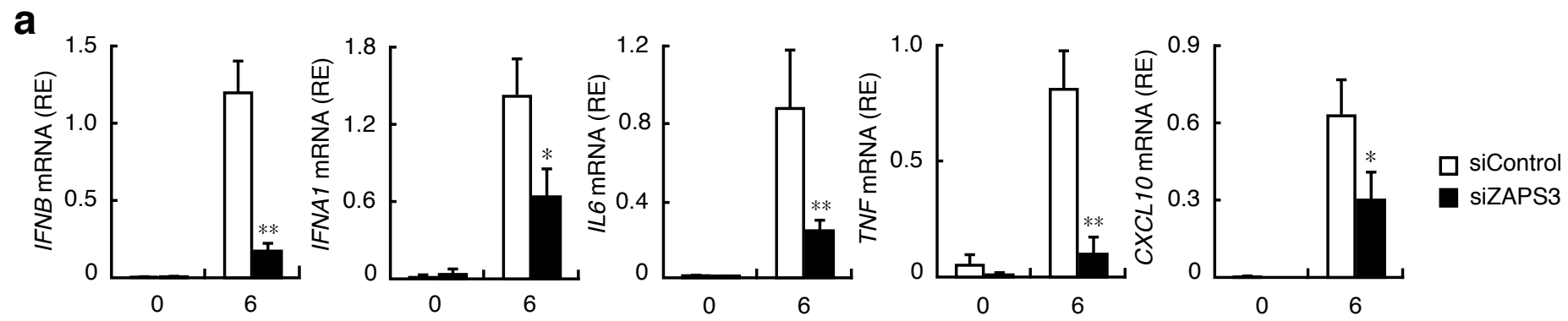

Time after infection $(\mathrm{h})$

Time after infection (h)

Time after infection (h)

Time after infection (h)

Time after infection (h)

b

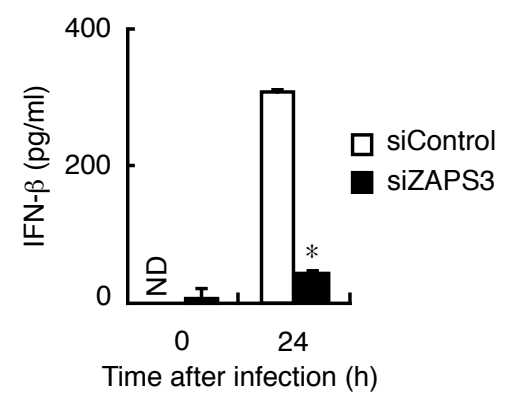

e

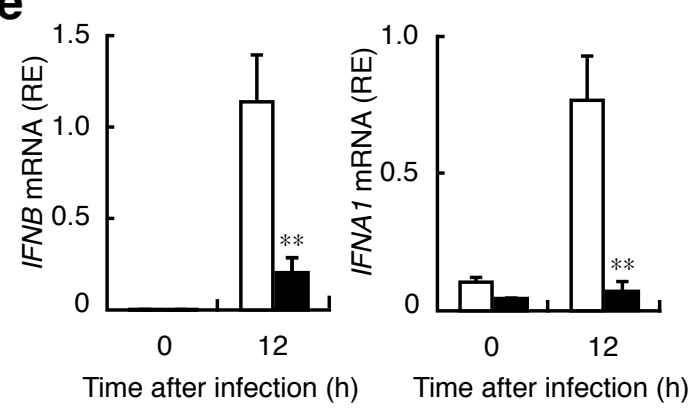

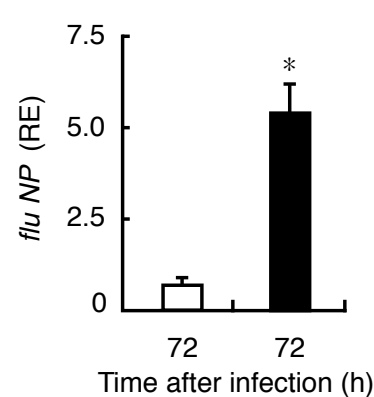

f

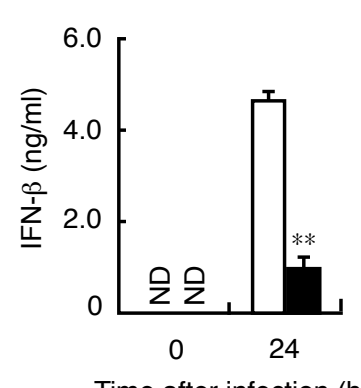

Time after infection (h)
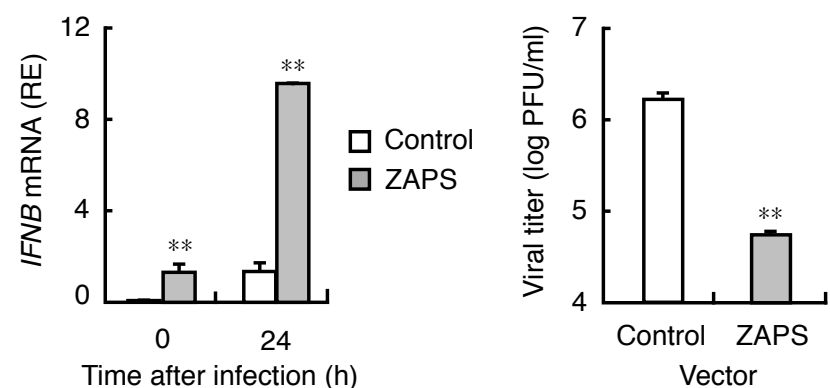

g

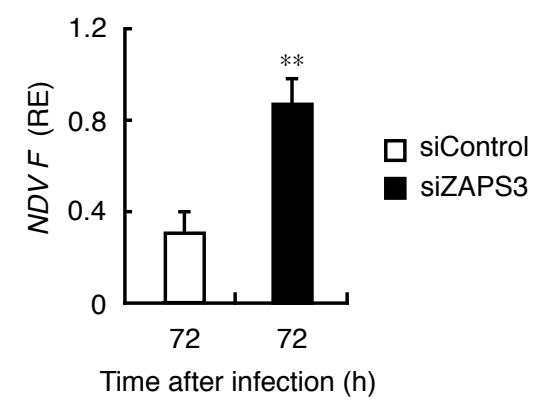

Fig. 5 


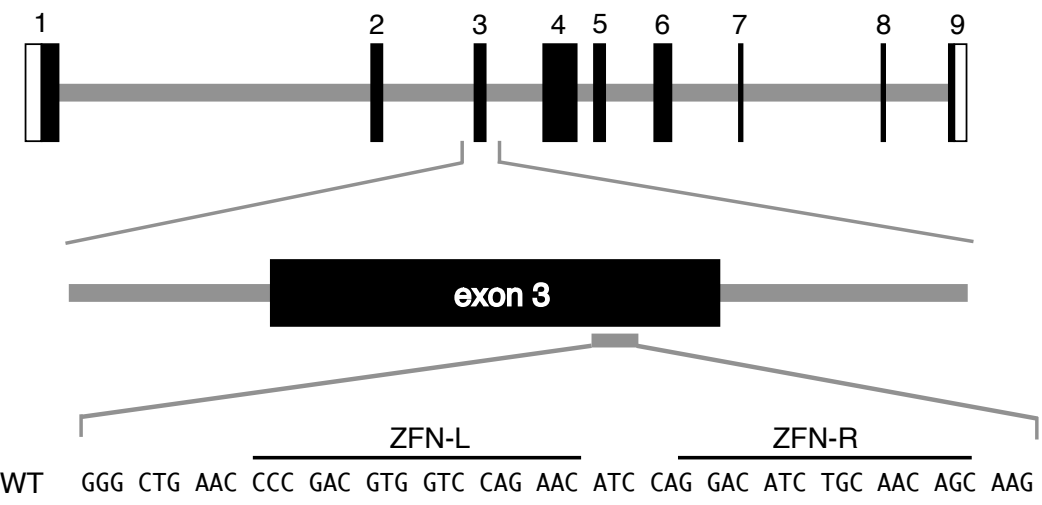

IP: anti-ZCCHV Blot: anti-ZCCHV

Blot: anti- $\beta$-actin (WCL)

C
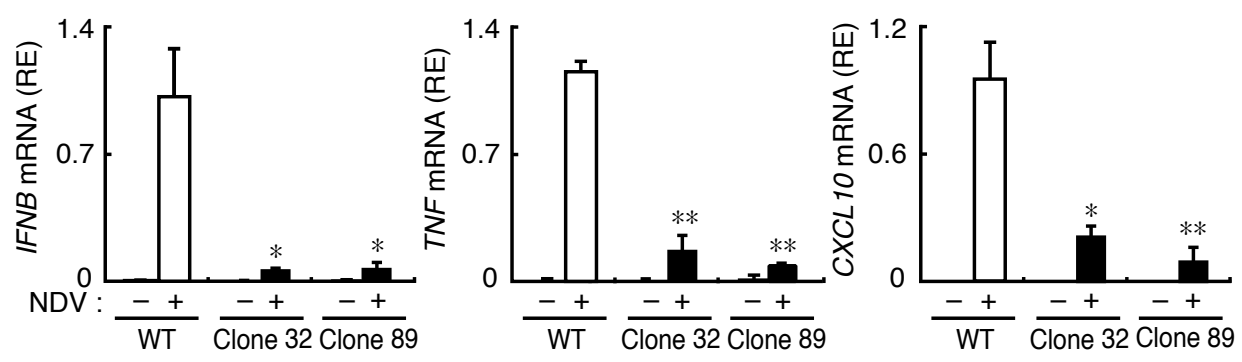

d

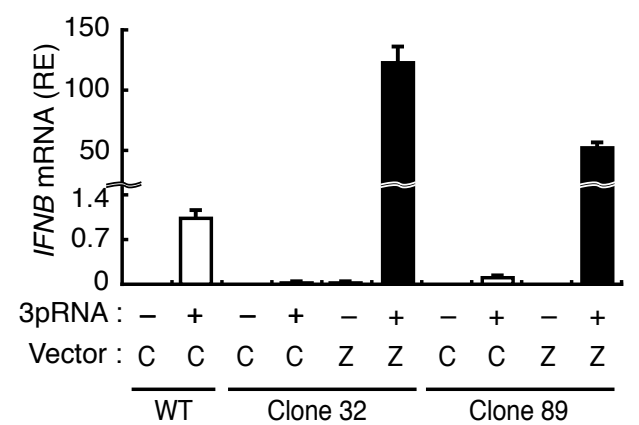

Fig. 6 